\title{
Antibiotic resistance of Escherichia coli and Salmonella enterica isolated from cabbage and lettuce samples in Tamale metropolis of Ghana
}

Frederick Adzitey

\begin{abstract}
Background: Contamination of vegetables by multidrug resistant pathogens is of global concern. This is the first report on the antibiotic resistance of Escherichia coli and Salmonella enterica from lettuce and cabbage sources in the Tamale metropolis of Ghana.

Method: A total of 26 Escherichia coli and 36 Salmonella enterica isolates were screened against nine antibiotics using the disc diffusion method.

Results: The overall resistance (R), intermediate (I) and susceptibility (S) of Escherichia coli $(n=11)$ from the cabbages were $54.55 \%, 9.09 \%$ and $36.36 \%$, respectively. Escherichia coli from cabbage samples were resistant to ofloxacin (100\%), ampicillin (90.01\%) and erythromycin (81.82\%). The overall resistance, intermediate and susceptibility of Escherichia coli $(n=15)$ from lettuce samples were $52.59 \%, 10.37 \%$ and $37.04 \%$, respectively. The isolates were also highly resistant to ofloxacin (100\%), erythromycin (93.33\%) and ampicillin (86.67\%). The resistant patterns AmpOfxE, CroAmpTeOfxEC and AmpTeOfxEC (exhibited by 3 isolates each) were the commonest pattern among the Escherichia coli isolates. Overall resistance, intermediate and susceptibility of Salmonella enterica $(n=18)$ from cabbages were $48.77 \%, 11.11 \%$ and $40.12 \%$, respectively. Salmonella enterica from cabbage samples were resistant to Ofloxacin (100\%) and Erythromycin (94.4\%). Overall resistance, intermediate and susceptibility of Salmonella enterica $(n=18)$ from lettuces were $53.70 \%, 10.49 \%$ and $53.70 \%$, respectively. Lettuce Salmonella enterica isolates were also highly resistant to Ofloxacin (100\%) and Erythromycin (94.4\%). The resistant pattern AmpOfxE was the commonest among the Salmonella enterica isolates and was exhibited by 12 isolates. Multiple antibiotic index (MAR index) ranged from 0.33 to 0.67 for the Escherichia coli isolates. MAR index for Salmonella enterica also ranged from 0.22 to 0.78 .

Conclusion: This study revealed that cabbage and lettuce samples in the Tamale metropolis are contaminated with antibiotic resistant Escherichia coli and Salmonella enterica.
\end{abstract}

Keywords: Antibiotics, Ghana, Escherichia coli, Salmonella enterica, Vegetables

(c) The Author(s). 2018 Open Access This article is distributed under the terms of the Creative Commons Attribution 4.0 International License (http://creativecommons.org/licenses/by/4.0/), which permits unrestricted use, distribution, and reproduction in any medium, provided you give appropriate credit to the original author(s) and the source, provide a link to the Creative Commons license, and indicate if changes were made. 


\section{Background}

Salmonella enterica and some strains of Escherichia coli are important foodborne pathogens. They cause Salmonella and Escherichia coli infections in humans. In the United States of America, Salmonella was the second most common cause of foodborne diseases, accounting for 149 (34\%) outbreaks and 3944 (39\%) illnesses, followed by Shiga toxin-producing Escherichia coli, which caused 27 (6\%) outbreaks and 302 (3\%) illnesses (Centers for Disease Control and Prevention (CDC) 2015). The most outbreak-associated illnesses were linked to seeded vegetables (e.g. tomatoes and pepper, 1121 illnesses), pork (924 illnesses), vegetable row crops (e.g. leafy vegetables, 383 illnesses), and chicken (333 illnesses) (CDC 2015).

In the European Union (EU) a total of 94,625 confirmed Salmonella infection cases were reported, representing a notification rate of 21.2 cases per 100,000 population (European Food Safety Authority (EFSA) and European Centre for Disease Prevention and Control (ECDC) 2016). The overall prevalence of Salmonella in animal- and vegetable-derived feed material was $5.13 \%$ (EFSA and ECDC, 2016). In addition, 5901 confirmed cases of shiga toxin-producing Escherichia coli (STEC) infections were reported in the EU, resulting in a notification rate of 1.27 cases per 100,000 population (EFSA and ECDC, 2016).

Various Salmonella serovars and Escherichia coli strains are responsible for these outbreaks. EFSA and ECDC (2016) reported that the two most commonly reported Salmonella serovars responsible for outbreaks in Europe were Salmonella Enteritidis (45.7\% outbreaks) and Salmonella Typhimurium (15.8\%). Salmonella Newport (3 outbreaks), Salmonella Enteritidis (2), Salmonella Paratyphi B (2), among others have been implicated in outbreaks caused by Salmonella in the USA (CDC 2015). The most commonly reported STEC serogroup in Europe was O157 (41.7\%), followed by serogroups O26, O103, O91, O145, O146 and O128 (EFSA and ECDC 2016). Escherichia coli serogroups O157 (4 outbreaks), O103 (2), O26 (2), O145 (1), and multiple serogroups (1) have also been reported in the USA (CDC 2015). Zoonosis report in unavailable in Ghana due to lack of support by responsible authorities for those responsible for such reports.

One of the effective ways of controlling Escherichia coli and Salmonella infections, and it associated outbreaks and illnesses is by the use of antibiotics. Nonetheless, the use of antibiotics for therapeutic and other purposes have received much attention and is of global concern. This is so because of the continuous development of multidrug resistance pathogens to commercial antibiotics. A number of factors including antimicrobial resistances (AMR) genes, genetic environment, bacterial cell, bacterial population/microbiota, human and animal population, and external environment contribute to the selection and dissemination of antimicrobial resistances. To minimise antimicrobial use, a multifaceted integrated approach has been recommended (European Food Safety Authority (EFSA) and the European Medicines Agency (EMA) 2017). Consumption of vegetables containing resistant Salmonella and Escherichia coli isolates is a threat to public health and needs to be given attention especially in countries where such phenomena is possible and rampant. Contamination of ready-to-eat vegetable salads by microbes in the Tamale metropolis has been reported (Abakari et al. 2018). Also, contamination of mixed vegetables by total aerobic bacteria and Staphylococcus aureus but not Salmonella species in the Kumasi metropolis has been reported (Amoah 2014).

In Ghana, there are fewer reports on antibiotic resistance of foodborne pathogens. The few studies available have also concentrated much on foodborne pathogens isolated from animals and humans (Mills-Robertson et al. 2002; Awua-Boateng 2007; Nsoah, 2015; Donkor et al. 2012; Adzitey 2015a; Adzitey et al. 2015; Andoh et al. 2016). However, vegetables in Ghana, including cabbages and lettuces are important sources of resistant foodborne pathogens and should not be ignored. Data on the antibiotic resistance of Salmonella enterica and Escherichia coli in Tamale Metropolis is unavailable. Therefore, this study reports for the first time on the prevalence of antibiotic resistant Salmonella enterica and Escherichia coli in cabbage and lettuce samples in Tamale Metropolis of Ghana.

\section{Methods \\ Location of study}

The study was conducted in the Tamale Metropolis found in the Northern Region of Ghana. The Metropolis is the capital town of the Northern Region and Ghana's fourth-largest city (Anonymous, 2018). It is found within the Guinea Savannah belt (Adzitey et al. 2016). The Metropolis is located on latitude $09^{\circ} 24^{\prime} 27^{\prime \prime}$ North and longitude $00^{\circ} 51^{\prime} 12$ " West, and occupies an area of approximately $750 \mathrm{~km}^{2}$ (Anonymous, 2018). Tamale is the third most populous settlement in Ghana, with a total population of 537,986 people and a population density of 480.77/ $\mathrm{km}^{2}$ (Anonymous, 2018; Adzitey et al. 2016). The daily mean temperature $\left({ }^{\circ} \mathrm{C}\right)$, average precipitation $\mathrm{mm}$ (inches) and average relative humidity (\%) are 27.9, 1090 and 60, respectively (Anonymous, 2018).

\section{Antimicrobial susceptibility of Salmonella species}

The disk diffusion method of Bauer-Kirby (1966) was used to determine the antibiotic resistance of 11 Escherichia coli from cabbage samples, 15 Escherichia coli from lettuce samples, 18 Salmonella enterica from cabbage samples and 18 Salmonella enterica from lettuce samples against nine different antibiotics. The nine antibiotics were Ampicillin (Amp) $30 \mu \mathrm{g}$ (Penicillin); Chloramphenicol (C) $30 \mu \mathrm{g}$ (Chloramphenicol); Ciprofloxacin (Cip) $5 \mu \mathrm{g}$ (Quinolones); Ceftriaxone (Cro) $30 \mu \mathrm{g}$ (Cephalosporin); Gentamicin (Cn) 
$10 \mu \mathrm{g}$ (Aminoglycoside); Erythromycin (E) $15 \mu \mathrm{g}$ (Macrolide); Ofloxacin (Ofx) $5 \mu \mathrm{g}$ (Quinolones); Suphamethoxazole/trimethoprim (Sxt) $22 \quad \mu \mathrm{g}$ (Sulfonamides) and Tetracycline (Te) $30 \mu \mathrm{g}$ (Tetracycline). All the antibiotic discs were purchased from Oxoid Limited, Basingstoke, United Kingdom.

Pure cultures of Escherichia coli and Salmonella enterica were grown overnight in Tryptic Soy Broth (Oxoid Limited, Basingstoke, United Kingdom) at $37{ }^{\circ} \mathrm{C}$ and the concentration adjusted using sterile Tryptic Soy Broth until a 0.5 McFarland turbidity was attained. One hundred $\mu \mathrm{l}$ of the culture was then swabbed onto Mueller Hinton agar (Oxoid Limited, Basingstoke, United Kingdom) using a sterile cotton swab. Three antimicrobial disks were placed on the surface of the agar plate at a distance to avoid overlapping of inhibition zones. The plates were incubated at $37^{\circ} \mathrm{C}$ for 16 to $18 \mathrm{~h}$ and the results were interpreted as sensitive, intermediate or resistance according to Clinical and Laboratory Standards Institute guidelines (Clinical and Laboratory Standards Institute, 2006). Escherichia coli ATCC 25922 and Salmonella enterica ATCC 14028 were used as controls for the antimicrobial susceptibility tests.

The multiple antibiotic resistance (MAR) index was calculated and interpreted according to Krumperman (1983) using the formula: $a / b$, where 'a' represents the number of antibiotics to which a particular isolate was resistant and ' $\mathrm{b}$ ' the total number of antibiotics tested.

\section{Results}

\section{Antimicrobial susceptibility of Escherichia coli from cabbage and lettuce sources}

The antimicrobial susceptibility of Escherichia coli from cabbage and lettuce samples is shown in Table 1. From Table 1, Escherichia coli of lettuce sources were highly resistant to ampicillin (86.67\%), erythromycin (93.33\%) and ofloxacin (100\%). Susceptibility between 60 and $70 \%$ was observed for ciprofloxacin, gentamicin, tetracycline and suphamethoxazole/trimethoprim. Intermediate resistance was $20 \%$ for ciprofloxacin and gentamicin. Escherichia coli isolates from cabbage samples were also highly resistant to ampicillin (90.91\%), erythromycin (81.82\%) and ofloxacin (100\%) but susceptible to ciprofloxacin (90.91\%). Intermediate resistances of $36.36 \%, 18.18 \%$ and $18.18 \%$ were observed for gentamicin, erythromycin and suphamethoxazole/trimethoprim, respectively. None of the Escherichia coli from lettuce and cabbage sources was susceptible to erythromycin and ofloxacin.

The antimicrobial resistance profile and MAR index of the Escherichia coli isolates is showed in Table 2. All Escherichia coli isolates from lettuce and cabbage samples were resistant to at least 3 antibiotics. The resistant patterns AmpOfxE (MAR index =0.33), AmpTeOfxESxt (MAR index $=0.56)$ and CroAmpOfxEC (MAR index = 0.56) were the commonest among the lettuce Escherichia coli isolates. These patterns were exhibited by two isolates each. With regards to cabbage Escherichia coli isolates, the resistant pattern CroAmpTeOfxEC with MAR index of 0.67 was the commonest. This pattern was exhibited by 3 isolates. One isolate from cabbage source was resistant to as many as seven different antibiotics (CroAmpTeOfxECSxt; MAR index of 0.78).

\section{Antimicrobial susceptibility of Salmonella enterica from cabbage and lettuce sources}

Most of the Salmonella enterica isolates from lettuce were resistant to ampicillin (72.22\%), erythromycin (94.44\%) and ofloxacin (100\%) (Table 3). Most of them were also susceptible to chloramphenicol (77.78\%), ciprofloxacin (94.44\%), ceftriaxone $(83.33 \%)$, tetracycline $(72.22 \%)$ and suphamethoxazole/trimethoprim (94.44\%). Intermediate resistances to gentamicin (50.00\%) and ampicillin (22.22\%) occurred. Majority of Salmonella enterica of cabbage sources were also resistant to ampicillin (72.22\%), erythromycin (94.44\%) and ofloxacin (100\%) but susceptible to ciprofloxacin $(72.22 \%)$ and Suphamethoxazole/trimethoprim

Table 1 Percentage antibiotic resistance of Escherichia coli from cabbage and lettuce samples

\begin{tabular}{|c|c|c|c|c|c|c|c|c|}
\hline \multirow[b]{2}{*}{ Antimicrobial } & \multirow[b]{2}{*}{${ }^{a} n / 15$} & \multirow[b]{2}{*}{ R (\%) } & \multicolumn{2}{|c|}{ E.coli from lettuce } & \multicolumn{4}{|c|}{ E.coli from cabbage } \\
\hline & & & I (\%) & S (\%) & $a_{n} / 11$ & $\mathrm{R}(\%)$ & I (\%) & $\mathrm{S}(\%)$ \\
\hline Ampicillin (Amp) $30 \mu \mathrm{g}$ & 13 & 86.67 & 13.33 & 0.00 & 10 & 90.91 & 0.00 & 9.09 \\
\hline Chloramphenicol (C) $30 \mu \mathrm{g}$ & 7 & 46.67 & 13.33 & 40.00 & 7 & 63.64 & 0.00 & 36.36 \\
\hline Ciprofloxacin (Cip) $5 \mu \mathrm{g}$ & 2 & 13.33 & 20.00 & 66.67 & 0 & 0.00 & 9.09 & 90.91 \\
\hline Ceftriaxone (Cro) $30 \mu \mathrm{g}$ & 6 & 40.00 & 13.33 & 46.67 & 6 & 54.55 & 0.00 & 45.45 \\
\hline Gentamicin (Cn) $10 \mu \mathrm{g}$ & 3 & 20.00 & 20.00 & 60.00 & 1 & 9.09 & 36.36 & 54.55 \\
\hline Erythromycin (E) $15 \mu \mathrm{g}$ & 14 & 93.33 & 6.67 & 0.00 & 9 & 81.82 & 18.18 & 0.00 \\
\hline Ofloxacin (Ofx) $5 \mu \mathrm{g}$ & 15 & 100.00 & 0.00 & 0.00 & 11 & 100.00 & 0.00 & 0.00 \\
\hline Suphamethoxazole/trimethoprim (Sxt) $22 \mu \mathrm{g}$ & 6 & 40.00 & 0.00 & 60.00 & 3 & 27.27 & 18.18 & 54.55 \\
\hline Tetracycline (Te) $30 \mu \mathrm{g}$ & 5 & 33.33 & 6.67 & 60.00 & 7 & 63.64 & 0.00 & 36.36 \\
\hline
\end{tabular}

${ }^{a} n$ number of resistant Escherichia coli, $S$ susceptible, I Intermediate, $R$ resistant 
Table 2 Antibiotic resistance profile and multiple antibiotic resistance index (MAR index) of individual Escherichia coli from cabbage and lettuce sources

\begin{tabular}{|c|c|c|c|c|c|c|c|}
\hline \multicolumn{4}{|c|}{ Cabbage } & \multicolumn{4}{|c|}{ Lettuce } \\
\hline Code & Antibiotic resistant profile ${ }^{a}$ & Number of antibiotics & $\overline{\text { MAR index }}$ & Code & Antibiotic resistant profile ${ }^{a}$ & Number of antibiotics & $\overline{\text { MAR index }}$ \\
\hline$\overline{\mathrm{EC}} 1$ & AmpOfxE & 3 & 0.33 & EL1 & AmpOfxE & 3 & 0.33 \\
\hline EC2 & AmpOfxESxt & 4 & 0.44 & EL2 & AmpOfxE & 3 & 0.33 \\
\hline EC3 & AmpTeOfxEC & 5 & 0.56 & EL3 & AmpOfxECnC & 5 & 0.56 \\
\hline EC4 & AmpTeOfxESxt & 5 & 0.56 & EL4 & AmpTeOfxE & 4 & 0.44 \\
\hline EC5 & CroAmpOfx & 3 & 0.33 & EL5 & AmpTeOfxECnC & 6 & 0.67 \\
\hline EC6 & CroAmpOfxEC & 5 & 0.56 & EL6 & AmpTeOfxECSxt & 6 & 0.67 \\
\hline EC7 & CroAmpTeOfxEC & 6 & 0.67 & EL7 & AmpTeOfxESxt & 5 & 0.56 \\
\hline EC8 & CroAmpTeOfxEC & 6 & 0.67 & EL8 & AmpTeOfxESxt & 5 & 0.56 \\
\hline EC9 & CroAmpTeOfxEC & 6 & 0.67 & EL9 & CroAmpOfx & 3 & 0.33 \\
\hline EC10 & CroAmpTeOfxECSxt & 7 & 0.78 & EL10 & CroAmpOfxEC & 5 & 0.56 \\
\hline \multirow[t]{5}{*}{ EC11 } & TeOfxCnC & 4 & 0.44 & EL11 & CroAmpOfxEC & 5 & 0.56 \\
\hline & & & & EL12 & CroAmpOfxECSxt & 6 & 0.67 \\
\hline & & & & EL13 & CroAmpOfxESxt & 5 & 0.56 \\
\hline & & & & EL14 & CroOfxEC & 4 & 0.44 \\
\hline & & & & EL15 & OfxECnSxt & 4 & 0.44 \\
\hline
\end{tabular}

${ }^{\mathrm{a} A m p i c i l l i n ~(A m p) ~} 30 \mu \mathrm{g}$; Chloramphenicol (C) $30 \mu \mathrm{g}$; Ciprofloxacin (Cip) $5 \mu \mathrm{g}$; Ceftriaxone (Cro) $30 \mu \mathrm{g}$; Gentamicin (Cn) $10 \mu \mathrm{g}$; Erythromycin (E) $15 \mu \mathrm{g}$; Ofloxacin (Ofx) $5 \mu \mathrm{g}$; Suphamethoxazole/trimethoprim (Sxt) $22 \mu \mathrm{g}$ and Tetracycline (Te) $30 \mu \mathrm{g}$

(83.33\%). Intermediate resistance was relatively high for ceftriaxone (38.89\%) and gentamicin (33.33\%).

Table 4 shows the antimicrobial resistance profile and MAR index of the Salmonella enterica isolates from lettuce and cabbage samples. All Salmonella enterica isolates from lettuce and cabbage samples were resistant to at least 2 antibiotics. The resistant pattern AmpOfxE (MAR index = 0.33) was the commonest among the Salmonella enterica of lettuce source and was exhibited by nine isolates. This was followed by the resistant pattern OfxE (MAR index = 0.22 ), which was showed by four isolates. Resistance to as many as seven (CroAmpTeOfxECSxt; MAR index = 0.78) and six (AmpTeOfxCipEC; MAR index $=0.67$ ) different antibiotics was exhibited by one Salmonella enterica each from lettuce source. Similarly, to Salmonella enterica from lettuce source, the resistant pattern AmpOfxE (MAR index $=0.33$ ) was the commonest among cabbage Salmonella enterica isolates. This was followed by the resistant patterns AmpTeOfxCipEC (MAR index = 0.67), AmpTeOfxEC (MAR index $=0.56$ ), OfxE (MAR index $=$ 0.22 ) and OfxECn (MAR index $=0.33$ ), which were exhibited by two isolates each. Resistance to as many as seven

Table 3 Percentage antibiotic resistance of Salmonella enterica from lettuce and cabbage samples

\begin{tabular}{|c|c|c|c|c|c|c|c|c|}
\hline \multirow[t]{2}{*}{ Antimicrobial } & \multicolumn{4}{|c|}{ Salmonella enterica from lettuce } & \multicolumn{4}{|c|}{ Salmonella enterica from cabbage } \\
\hline & $a_{n} / 18$ & $\mathrm{R}(\%)$ & I (\%) & $\mathrm{S}(\%)$ & ${ }^{a} \mathrm{n} / 18$ & $\mathrm{R}(\%)$ & I (\%) & S (\%) \\
\hline Ampicillin (Amp) $30 \mu \mathrm{g}$ & 13 & 72.22 & 22.22 & 5.56 & 13 & 72.22 & 16.67 & 11.11 \\
\hline Chloramphenicol (C) $30 \mu \mathrm{g}$ & 3 & 16.67 & 5.56 & 77.78 & 10 & 55.56 & 5.56 & 38.89 \\
\hline Ciprofloxacin (Cip) $5 \mu \mathrm{g}$ & 1 & 5.56 & 0.00 & 94.44 & 4 & 22.22 & 5.56 & 72.22 \\
\hline Ceftriaxone (Cro) $30 \mu \mathrm{g}$ & 1 & 5.56 & 11.11 & 83.33 & 0 & 27.78 & 38.89 & 61.11 \\
\hline Gentamicin (Cn) $10 \mu \mathrm{g}$ & 0 & 0.00 & 50.00 & 50.00 & 5 & 20.00 & 33.33 & 38.89 \\
\hline Erythromycin (E) $15 \mu \mathrm{g}$ & 17 & 94.44 & 0.00 & 5.56 & 17 & 94.44 & 0.00 & 5.56 \\
\hline Ofloxacin (Ofx) $5 \mu \mathrm{g}$ & 18 & 100.00 & 0.00 & 0.00 & 18 & 100.00 & 0.00 & 0.00 \\
\hline Suphamethoxazole/trimethoprim (Sxt) $22 \mu \mathrm{g}$ & 1 & 5.56 & 0.00 & 94.44 & 3 & 16.67 & 0.00 & 83.33 \\
\hline Tetracycline (Te) $30 \mu \mathrm{g}$ & 4 & 22.22 & 5.56 & 72.22 & 9 & 50.00 & 0.00 & 50.00 \\
\hline
\end{tabular}

${ }^{a} n$ number of resistant Salmonella enterica, $S$ susceptible, $I$ Intermediate, $R$ resistant 
Table 4 Antibiotic resistance profile and multiple antibiotic resistance index (MAR index) of individual Salmonella enterica from cabbage and lettuce sources

\begin{tabular}{|c|c|c|c|c|c|c|c|}
\hline \multicolumn{4}{|c|}{ Lettuce } & \multicolumn{4}{|c|}{ Cabbage } \\
\hline Code & Antibiotic resistant profile ${ }^{a}$ & Number of antibiotics & $\overline{\text { MAR index }}$ & Code & Antibiotic resistant profile $e^{a}$ & Number of antibiotics & $\overline{\text { MAR index }}$ \\
\hline$\overline{\mathrm{L} 1}$ & AmpOfx & 2 & 0.22 & C1 & AmpOfx & 2 & 0.22 \\
\hline L2 & AmpOfxE & 3 & 0.33 & $C 2$ & AmpOfxCipEC & 5 & 0.56 \\
\hline L3 & AmpOfxE & 3 & 0.33 & C3 & AmpOfxE & 3 & 0.33 \\
\hline L4 & AmpOfxE & 3 & 0.33 & C4 & AmpOfxE & 3 & 0.33 \\
\hline L5 & AmpOfxE & 3 & 0.33 & C5 & AmpOfxE & 3 & 0.33 \\
\hline L6 & AmpOfxE & 3 & 0.33 & C6 & AmpTeOfxCipEC & 6 & 0.67 \\
\hline L7 & AmpOfxE & 3 & 0.33 & C7 & AmpTeOfxCipEC & 6 & 0.67 \\
\hline L8 & AmpOfxE & 3 & 0.33 & C8 & AmpTeOfxCipECnC & 7 & 0.78 \\
\hline L9 & AmpOfxE & 3 & 0.33 & C9 & AmpTeOfxCipECSxt & 7 & 0.78 \\
\hline L10 & AmpOfxE & 3 & 0.33 & $\mathrm{C} 10$ & AmpTeOfxEC & 5 & 0.56 \\
\hline L11 & AmpTeOfxCipEC & 6 & 0.67 & C11 & AmpTeOfxEC & 5 & 0.56 \\
\hline L12 & AmpTeOfxEC & 4 & 0.44 & $\mathrm{C} 12$ & AmpTeOfxECnC & 6 & 0.67 \\
\hline L13 & CroAmpTeOfxECSxt & 7 & 0.78 & C13 & AmpTeOfxECSxt & 6 & 0.67 \\
\hline L14 & OfxE & 2 & 0.22 & C14 & OfxE & 2 & 0.22 \\
\hline L15 & OfxE & 2 & 0.22 & C15 & OfxE & 2 & 0.22 \\
\hline L16 & OfxE & 2 & 0.22 & C16 & OfxECn & 3 & 0.33 \\
\hline L17 & OfxE & 2 & 0.22 & $\mathrm{C} 17$ & OfxECn & 3 & 0.33 \\
\hline L18 & TeOfxE & 3 & 0.33 & $\mathrm{C} 18$ & TeOfxECnCSxt & 6 & 0.67 \\
\hline
\end{tabular}

${ }^{a}$ Ampicillin (Amp) $30 \mu$; Chloramphenicol (C) $30 \mu$; Ciprofloxacin (Cip) $5 \mu$; Ceftriaxone (Cro) $30 \mu g$; Gentamicin (Cn) $10 \mu g$; Erythromycin (E) $15 \mu$; Ofloxacin (Ofx) $5 \mu \mathrm{g}$; Suphamethoxazole/trimethoprim (Sxt) $22 \mu \mathrm{g}$ and Tetracycline (Te) $30 \mu \mathrm{g}$

different antibiotics (AmpTeOfxCipECnC; MAR index = 0.78 and AmpTeOfxCipECSxt; MAR index =0.78) was exhibited by two isolates.

\section{Discussion}

This study provides data for the first time on the prevalence of resistant Escherichia coli and Salmonella enterica from lettuce and cabbages sources in the Tamale metropolis of Ghana. It also provides baseline information by which other studies can be compared. Resistance of foodborne pathogens including Escherichia coli and Salmonella enterica to multiple antibiotics has become an emerging public health issue worldwide. The use of antibiotics in agricultural practices have contributed immensely to the development of resistant foodborne pathogens (Cohen 2000; Adams and Moss 2008; Golly et al. 2016; Jongman and Korsten 2016). Antibiotics are mostly used for treatment of animals and humans against bacterial infections. This can lead to some bacteria developing resistance against the antibiotics being used to control them. Subsequently, animals and humans share these pathogens which find their way into water bodies some of which are used to irrigate vegetables. The results of this is that vegetables get contaminated with these resistant pathogens which can also be easily transferred to other food sources. A number of sources including application of manures to the farm from slaughter houses, in vitro propagation of crops (tissue cultured plants), antibiotics spray on the crops in the orchard, soil and water contamination with fecal material and effluent from farm animals at the field, and genetic engineering causing increased antibiotic resistance have noted as sources by which antibiotic resistances are incorporation into fruits and vegetables (Rashmi et al. 2017). In Nigeria, Afolabi and Oloyede (2010) found irrigation water as possible source of food borne pathogens in raw vegetables. In South Africa, Jongman and Korsten (2016) indicated that there is a link between the Escherichia coli isolates from irrigation water sources and leafy green vegetables in their phenotypic (antimicrobial) and genotypic (DNA fingerprinting) analyses. Schwaiger et al. (2011) suggested that expressing resistance by bacteria isolated from vegetables is at the expense of bacterial viability, since vegetables purchased directly at the farm are probably fresher than at the supermarket, and they have not been exposed to stress factors. Maka and Popowska (2016) reported that the use of a single antibiotic might result in the development of resistance to other antimicrobial compounds of the same or different classes.

Antibiotic resistance of Escherichia coli isolates of lettuce and cabbage samples in the present study is comparable. The overall resistance (54.55\% vs $52.59 \%$ ), intermediate resistance (9.09\% vs $10.37 \%)$ and susceptibility $(36.36 \%$ vs $37.04 \%$ ) for cabbage and lettuce, respectively were similar. 
Rodloff et al. (2008) indicated that intermediate resistance is associated with an uncertain therapeutic effect. Escherichia coli from both sources showed high resistance to the penicillin (ampicillin), macrolide (erythromycin) and ofloxacin (quinolone) but showed susceptibility to the quinolone (ciprofloxacin). Susceptibility to the cephalosporin (ceftriaxone), aminoglycoside (gentamicin) and sulfonamide (suphamethoxazole/trimethoprim) were also similar among the Escherichia coli lettuce and cabbage isolates. Intermediate resistances were more prevalent in Escherichia coli of cabbage source than that of lettuce ( 5 vs 3, respectively). Golly et al. (2016) reported that Escherichia coli from cabbage, lettuce and carrot sources were resistant to ampicillin (90.9\%), tetracycline $(54.5 \%)$, gentamicin $(18.2 \%)$, chloramphenicol (63.6\%) and ceftriaxone (54.5\%), which were relatively similar to that of the present study. Contrarily to this study, Jongman and Korsten (2016) found that Escherichia coli isolates from green leafy vegetables were susceptible to ceftriaxone $(100 \%)$ and gentamicin (100\%).

All Escherichia coli isolates from lettuce and cabbage samples were resistant to at least 3 different antibiotics, thus $100 \%$ multidrug resistant was observed among the Escherichia coli isolates. Furthermore, cabbage Escherichia coli isolates were resistant to seven (9.09\%), six (27.27\%), five (27.27\%) and four (18.18\%) different antibiotics, while lettuce Escherichia coli isolates were resistant to six (20\%), five $(40 \%)$ and four $(20 \%)$ different antibiotics. Jongman and Korsten (2016) found that 19 (14.6\%) of Escherichia coli isolates from leafy green vegetables $(n=70)$ and water $(n=60)$ sources were resistant to one antibiotic (tetracycline) and 92 (70.7\%) were resistant to various antibiotics (including ampicillin, cefoxitin, and nalidixic acid).

Antibiotic resistance of Salmonella enterica from lettuce and cabbage samples revealed some similarities and differences in resistance percentages and patterns. The overall resistance $(48.77 \%$ vs $35.80 \%)$, intermediate resistance $(11.11 \%$ vs $10.49 \%$ ) and susceptibility (40.12\% vs $53.70 \%$ ) for cabbage and lettuce, respectively were quite dissimilar. Resistance to the penicillin (ampicillin), macrolide (erythromycin) and quinolone (ofloxacin) were similar but not resistance to the chloramphenicol (chloramphenicol), quinolone (ciprofloxacin), cephalosporin (ceftriaxone), aminoglycoside (gentamicin), tetracycline (tetracycline) and sulfonamide (suphamethoxazole/trimethoprim). Susceptibility followed similar trend except for resistance to erythromycin. Intermediate resistances were also similar between lettuce and cabbage Salmonella enterica isolates. Intermediate resistance has been suggested to be those isolates which are not clearly resistant or susceptible, and such isolates have the tendency to easily become resistant (Adzitey et al. 2012; Adzitey et al. 2015). Generally, Salmonella enterica isolates from cabbage were more resistant than those from lettuce sources. Golly et al. (2016) found that Salmonella species isolated from cabbage, lettuce and carrot sources were resistant to ampicillin (100\%), tetracycline (63.6\%), gentamicin (9.1\%), chloramphenicol (72.7\%) and ceftriaxone (63.6\%), which was relatively dissimilar to the present study.

The lettuce and cabbage Salmonella enterica isolates were resistant to at least 2 different antibiotics. Resistance to seven (5.56\%), six (5.56\%), and four (5.56\%) different antibiotics occurred for lettuce Salmonella enterica isolates. Resistance to seven (11.11\%), six (22.78\%) and five (16.67\%) antibiotics was found for cabbage Salmonella enterica isolates. This indicates that cabbage and lettuce samples harboured high levels of multidrug resistant Salmonella enterica isolates. The resistance pattern ampicillin-ofloxacin-erythromycin (AmpOfxE) was the commonest considering all Salmonella enterica isolates of both lettuce and cabbage sources. Seven and twelve resistance patterns were found for lettuce and cabbage Salmonella enterica isolates, respectively. Golly et al. (2016) reported that their Salmonella isolates showed seven resistance patterns to eight commonly used antibiotics. They also reported that all their isolates exhibited multidrug resistance.

Antibiotics and their resistant patterns by foodborne pathogens have evolved over the years. Differences and similarities in resistance patterns can occur among foodborne isolates from the same or different sources. These differences have been reported to be widely due to the differences in in geographical locations, the bacteria species involved, the animal production systems employed, the extent to which antibiotics are used, sampling techniques and period of sampling (Adzitey 2015b). Rashmi et al. (2017) stated that, the presence of residual antibiotics in foods constitute an important health risk because of the increased microbial resistance detected in latest years.

\section{Conclusions}

This study revealed that lettuce and cabbage samples were contaminated with resistant Escherichia coli and Salmonella enterica isolates. This could be as a result of lettuces and cabbages getting in contact with contaminated water used for irrigation. It could also be due to these vegetables getting contact with contaminated soils they are planted on. There is the need to adhere to good production practices to help decrease the incidence of multidrug resistant Escherichia coli and Salmonella enterica strains. Furthermore, regular antibiotic susceptibility surveillance is required to track changes in resistant patterns. This work provides a first line data for antibiotic resistant patterns of Escherichia coli and Salmonella enterica from vegetables in the northern region of Ghana.

\section{Limitations}

Detection of genes in Escherichia coli and Salmonella enterica conferring resistance to the antibiotics examined would have been helpful to improve this study. However, this was not done due to lack of molecular equipment, reagents and the cost involved in carrying out such an experiment. 


\section{Abbreviations}

Amp: Ampicillin; C: Chloramphenicol; CDC: Centres for Disease Control and Prevention; Cip: Ciprofloxacin; Cn: Gentamicin; Cro: Ceftriaxone; E: Erythromycin; ECDC: European Centre for Disease Prevention and Control; EFSA: European Food Safety Authority; EU: European Union; MAR index: Multiple antibiotic index; ${ }^{\circ} \mathrm{C}$ : Degrees Celsius; Ofx: Ofloxacin; STEC: Shiga toxin-producing Escherichia coli; Sxt: Suphamethoxazole/ trimethoprim; Te: Tetracycline; $\mu$ : microlitre

\section{Acknowledgments}

The author is grateful to the University for Development Studies, for allowing him to use the Spanish Laboratory to carry out this experiment. The author is also grateful to Sabina for her contribution towards getting the isolates.

\section{Funding}

Self-funded.

\section{Availability of data and materials}

All datasets on which the conclusions of the manuscript rely are presented in the paper.

\section{Author's contribution}

FA designed, carried out the experiment and wrote the manuscript. The author read and approved the final manuscript.

\section{Authors' information}

Frederick Adzitey is an Associate Professor of the Department of Animal Science of the University for Development Studies. He has a PhD in Food Safety from Universiti Sains Malaysia and MSc in Meat Science and Technology from University of Bristol. His scientific interests cover a broad range of topics, including animal and meat science, food quality and safety, antibiotic resistance and molecular characterization of foodborne and waterborne pathogens.

\section{Ethics approval and consent to participate}

Not applicable.

\section{Consent for publication}

Not applicable.

\section{Competing interests}

The author declares that he/she has no competing interests.

\section{Publisher's Note}

Springer Nature remains neutral with regard to jurisdictional claims in published maps and institutional affiliations.

Received: 10 May 2018 Accepted: 23 August 2018

Published online: 11 September 2018

\section{References}

Abakari G, Cobbina SJ, Yeleliere E. Microbial quality of ready-to-eat vegetable salads vended in the central business district of tamale, Ghana. Int J Food Contam. 2018:5:1-9.

Adams MR, Moss MO. Food Microbiology. Royal Society of Chemistry Publishing, Cambridge, UK. ISBN-13: 9780854042845, 2008; Pages: 463.

Adzitey F. Antibiotic resistance of Escherichia coli isolated from beef and its related samples in Techiman municipality of Ghana. Asian J Anim Sci. 2015a;9:233-40.

Adzitey F. Antibiotic classes and antibiotic susceptibility of bacterial isolates from selected poultry; a mini review. World's Vet J. 2015b;5:36-41.

Adzitey F, Rusul G, Huda N, Cogan T, Corry J. Prevalence, antibiotic resistance and RAPD typing of Campylobacter species isolated from ducks, their rearing and processing environments in Penang, Malaysia. Int J Food Microbiol. 2012;154:197-205.

Adzitey F, Nsoah JK, Teye G. Prevalence and antibiotic susceptibility of Salmonella species isolated from beef and its related samples in Techiman municipality of Ghana. Turk J Agric Food Sci Technol. 2015;3:644-50.

Adzitey F, Ashiagbor CNK, Abu H. Prevalence and antibiotic susceptibility of Salmonella spp. from water sources in tamale, Ghana. Int J One Health. 2016;2:24-8.

Afolabi OR, Oloyede AR. (2010). Irrigation water as possible source of foodborne pathogens in raw vegetables. J Nat Sci Eng Technol 2010; 9:117-122.
Amoah D. Microbial risk assessment of mixed vegetable salads from selected canteens in the Kumasi Metropolis. A thesis submitted to the Department of Food Science and Technology, College of Science in partial fulfilment of the requirement for the degree of master of science (food quality management) Available from: http://ir.knust.edu.gh/xmlui/handle/123456789/7081; 2014.

Andoh LA, Dalsgaard A, Obiri-Danso K, Newman MJ. Prevalence and antimicrobial resistance of Salmonella serovars isolated from poultry in Ghana. Epidemiol Infect. 2016;144:3288-99.

Anonymous. Tamale Metropolis. Available from: https://en.wikipedia.org/wiki/ Tamale,_Ghana; 2018. Accessed 25 Apr 2018.

Awua-Boateng NA. Incidence of Salmonella bacteraemia and antibiotic resistance of Salmonella in paediatric patients in Kumasi. A Master's Thesis submitted to the Department of Clinical Microbiology, Kwame Nkrumah University of Science and Technology, Ghana; 2007.

Centers for Disease Control and Prevention (CDC). Surveillance for foodborne disease outbreaks, United States, 2015, Annual Report. Atlanta, Georgia: US Department of Health and Human Services. Available from: https://www.cdc.gov/foodsafety/pdfs/ 2015FoodBorneOutbreaks_508.pdf; 2017.

Clinical and Laboratory Standards Institute. Methods for antimicrobial dilution and disk susceptibility testing of infrequently isolated or fastidious bacteria; Approved Guideline (M45-A). Wayne: Clinical and Laboratory Standards Institute; 2006

Cohen ML. Changing patterns of infectious disease. Nature. 2000;406:762-7.

Donkor ES, Newman MJ, Yeboah-Manu D. Epidemiological aspects of nonhuman antibiotic usage and resistance: implications for the control of antibiotic resistance in Ghana. Trop Med Int Health. 2012;17:462-8.

European Food Safety Authority (EFSA) and European Centre for Disease Prevention and Control (ECDC). The European Union summary report on trends and sources of zoonoses, zoonotic agents and food-borne outbreaks in 2015. EFSA J. 2016;14:4634-865.

European Food Safety Authority (EFSA) and the European Medicines Agency (EMA) EMA and EFSA Joint Scientific Opinion on measures to reduce the need to use antimicrobial agents in animal husbandry in the European Union, and the resulting impacts on food safety (RONAFA). Available from: https://efsa.onlinelibrary.wiley.com/doi/10.2903/j.efsa.2017.4666; 2017.

Felix Mills-Robertson F, Addy ME, Mensah P, Crupper SS. Molecular characterization of antibiotic resistance in clinical Salmonella Typhi isolated in Ghana. FEMS Microbiol Lett. 2002;215:249-53.

Golly MK, Salifu PS, Mills-Robertson FC. Resistance of bacteria isolates from cabbage (Brassica oleracea), carrots (Daucus carota) and lettuce (Lactuca sativa) in the Kumasi metropolis of Ghana. Int J Nutr Food Sci. 2016;5:297-303.

Jongman M, Korsten L. Genetic diversity and antibiotic resistance of Escherichia coli isolates from different leafy green production systems. J Food Prot. 2016;79:1846-53.

Mąka $Ł$, Popowska M. Antimicrobial resistance of Salmonella spp. isolated from food. Rocz Panstw Zakl Hig. 2016;67:343-58.

Nsoah JK. Effect of postharvest handling on microbial and aethestic qualities of fresh beef in the Techiman municipality. A thesis submitted to the Department of Animal Science, University for Development Studies, in partial fulfillment of the requirements for the award of Masters of Philosophy Degree in Animal Science. 2015;1-162.

Rashmi HB, Bharti SK, Gogai M, Devi S, Anita Ganguly S. Antibiotic resistance: role of fruits and vegetables in the food basket. Int J Pure Appl Biosci. 2017;5:169-73.

Rodloff A, Bauer T, Ewig S, Kujath P, Müller E. Susceptible, intermediate and resistant - the intensity of antibiotic action. Dtsch Arztebl Int. 2008;105:657-62.

Schwaiger K, Helmke K, Hölzel C, Bauer J. Antibiotic resistance in bacteria isolated from vegetables with regards to the marketing stage (farm vs. supermarket). Int J Food Microbiol. 2011;148:191-6. 